\title{
Survey of Delay Tolerant Network - An Architectural Prospective
}

\author{
Lalitesh Kumar \\ Choudhary \\ CSE, UIT, RGPV \\ Bhopal, India
}

\author{
Murlidhar Prasad \\ Singh \\ CSE, UIT, RGPV \\ Bhopal, India
}

\author{
Uday Chaurasia \\ CSE, UIT, RGPV \\ Bhopal, India
}

\author{
Manish Kumar \\ Ahirwar \\ CSE, UIT, RGPV \\ Bhopal, India
}

\begin{abstract}
One of the major problems of delay tolerant network is the absence of complete path in between the source and destination. In the open literature, these networks are known as challenged network/Intermittently Connected Networks (ICNs). The existing protocols fail to operate properly in the context of intermittently connected network, which raises a variety of new challenging problems. This paper sheds the light over the architectural aspects of these networks. Here architectural aspects includes specification, working concept, required modification in traditional protocol, necessary included services etc. We have concentrated on basic mechanism of each of the above-mentioned topics and try to highlight some pending open issues.
\end{abstract}

\section{Keywords}

Delay-tolerant network, bundle protocol, DTN architecture.

\section{INTRODUCTION}

A delay tolerant network is an emerging network in which complete path from source to destination does not exist. Now a days various challenged or opportunistic networking application are being used such as wildlife monitoring [16], search and rescue system, underwater sensor network, vehicular ad-hoc network, data mule, packet switched network, underdeveloped region connectivity [7] and many others. The working of these networks is affected by various limiting factors such as High latency \&low data rate (10kbps under water), end to end node Disconnection Problem, Long message queuing Times, as well as Limited resources in terms of limited memory and processing capability which leads to the unstable path or connectivity in between two nodes in opportunistic network. As the contact schedules between two nodes are completely opportunistic, we cannot accurately predict the meeting probability or the contract schedule of the node. To deal with such adversity these networks makes the use of store carry and forward mechanism as well as a custodian retransmission mechanism.

The concept of Delay Tolerant network [3] makes the use of store carry and forward (SCF) mechanism in which first of all, the message to be sent to any node has to be stored or buffered in the form of bundle (basic unit).Then it is carried until it find some interesting node (may be on the basis of some utility values).And as a last step forward the particular message to that node. This process continues until the message is delivered to the destination or is dropped due to network congestion or on the basis of Time to live (TTL) values.

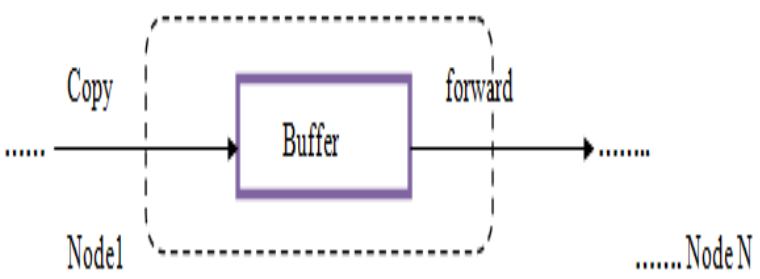

Figure 1: Store Carry \& Forward Mechanism

In traditional network TCP provides reliable data transfer services where only end nodes are responsible for acknowledgement of error free reception of packets. But in DTN scenario this is impossible, for example in wireless sensor network (WSN) a node has limited storage capacity and is unable to store packet for a long period of time to handle possible retransmission.

Due to this reason, the reliability is achieved in DTN by making use of custody transfer mechanism [6]. The concept of custody transfer can be defined as the data transfer mechanism, in which the responsibility of data segment (bundle or bundle fragment), migrates with the data as it progresses across a series of network hops for the purpose of reliable delivery on a hop-by-hop basis as compared to an end-to-end which is impractical over intermittently connected nodes and free up storage space for other newly incoming ones.

For achiving reliable communication over intermittently connected environment DTN adopts the delay tolerance capability of email facility like acknowledgement facility only on message failure as well as asynchronus message transmission. Here the main motivation behind this is that to efficiently utilize precious available bandwidth. To cope with network uncertainty, concept of late binding is used in which first the network region is identified and then the destination node is approached instead of binding destination node from source node at the time of packet generation.

Also DTN networking approach makes the use of message switching instead of packet switching mechanism as packet drop probability may reach up to maximum in challenging environment. 


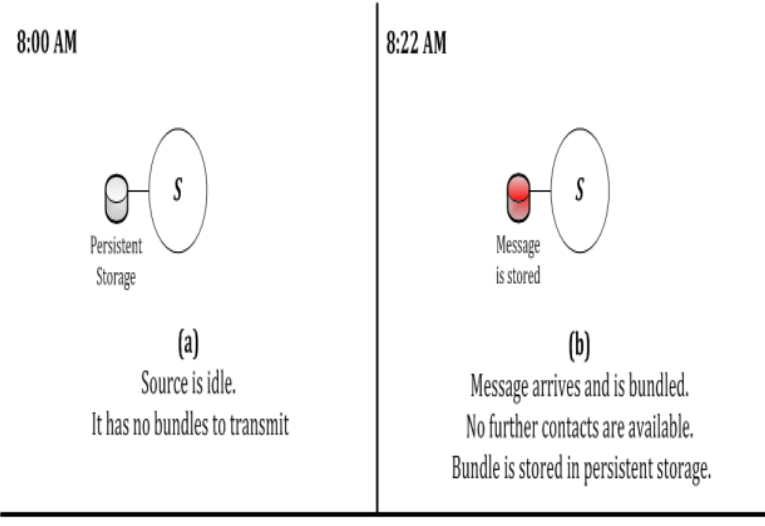

$8: 40$ AM

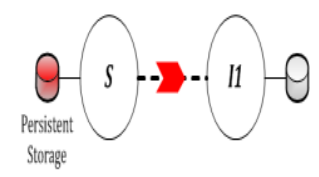

(c)

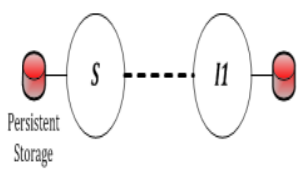

(d)
Sencounters 11 and transmits the bundle to it. $\quad$ I1 stores the bundle in its persistent storage.

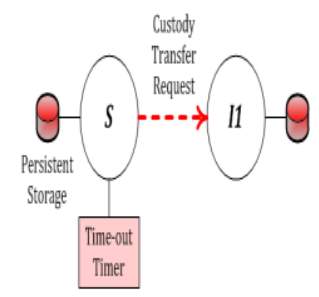

(e)

S requests custody transfer to 11 .

Sstarts a request time-out timer

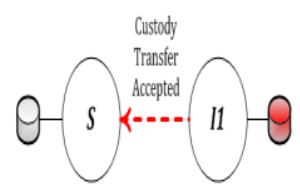

(f)

I1 acknowledges bundle's custody to S.

Sdeletes bundle from persistent storage.

Custody transfer mechanism completed.
Fig 2: Custody Retransmission Mechanism

To provide such mechanism, this network makes the use of the bundle protocol. The necessity of the Bundle Protocol lies in the fact/concept that all control signals and information data are combined in a single atomic entity, called bundle [4], which is transmitted across a delay tolerant network.

Although bundle protocol runs over TCP/IP, it also runs on other lower-layer protocols (so-called convergence layers which are equipped with a set of protocol-specific Convergence Layer adapters that are responsible of guiding DTN bundles through their corresponding protocol's different procedures) for example proprietary protocols deployed in sensor network as well as LTP [56] in deep-space [19] deployments. BP forms an overlay that employs persistent storage [26] to help combat network interruption and for its store and forward function.

The rest of the paper has been organized as follows. Section 2 gives a brief description of Architectural overview of delay tolerant network. Message transmission and the bundle protocol has been briefly explained in section 3. And finally section 4 includes some concluding remarks.

\section{ARCHITECTURAL OVERVIEW}

A delay tolerant network follow an overlay network architecture [5], used for performing communication among heterogeneous and different networks. As DTN is a partition based network, it tries to enable connectivity among disconnected network/part of the network.

The figure shown below demonstrates the previously explained concept of delay tolerant network. As shown, networks in each of the isolated regions may well connected or may intermittently connected. An

The intermittently connected regional network can be considered as a very special class as it can be taken under consideration as two separate regions using the same underlying technology. Here nodes from any region may disengage and result transfer to any other region. This results disruption of links as any transferring node get out of communication range of other nodes in the network.

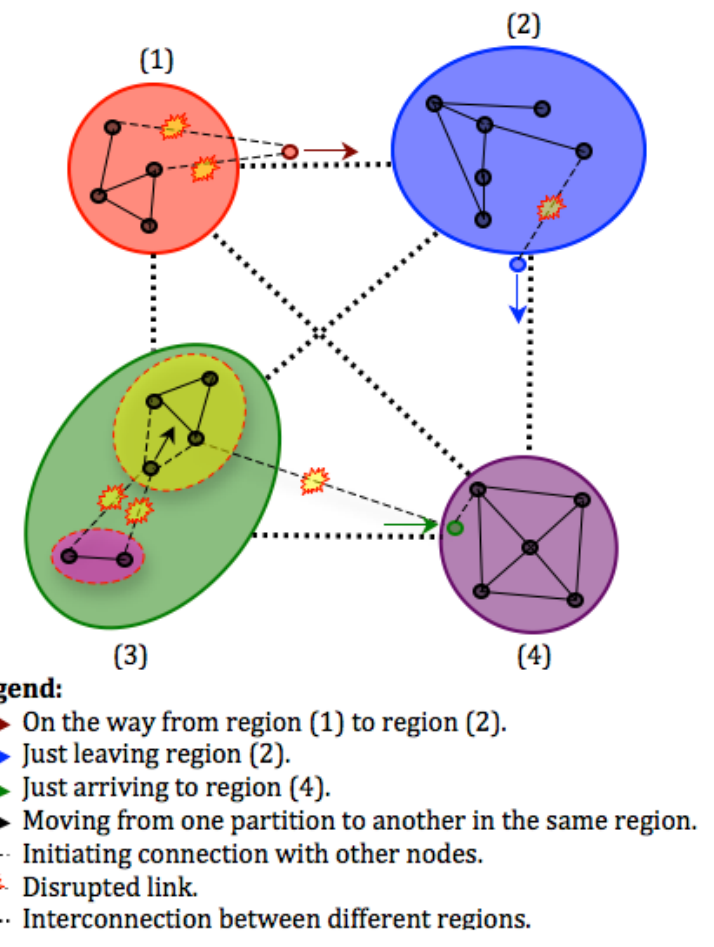

Fig 3: DTN Protocol Stack Communication Diagram

As we know the delay tolerant network makes use of two mechanisms in order to resist in challenged environment i.e. store carry and forward mechanism [16] as well as custody based retransmission. To support above we need a additional protocol. The bundle protocol is an application layer protocol that is used to construct a store-and-forward overlay network. It essentially runs at the application layer and generally follows the overlay-network approach.

Although the bundle protocol [8] runs over TCP/IP, it also runs on other lower-layer protocols (so-called convergence layers which are equipped with a set of protocol-specific Convergence Layer adapters that are responsible of guiding DTN bundles through their corresponding protocol's different procedures) for example proprietary protocols deployed in sensor network as well as LTP in deep-space deployments. BP forms an overlay that employs persistent storage to help combat network interruption and for its store and forward function. 
BP's key capabilities include custody-based retransmission; an ability to cope with intermittent connectivity; under which it takes the advantage of predicted, scheduled and opportunistic connectivity.

In an overlay network, there is no limit to the number of intermediate nodes as well as the number of gateways that may exist between two ultimate end points. The importance is that intermittent connectivity is also supported regardless of the underlying network types and the transportation of a bundle is presumably reliable between each and every one of the bundle layer instances.

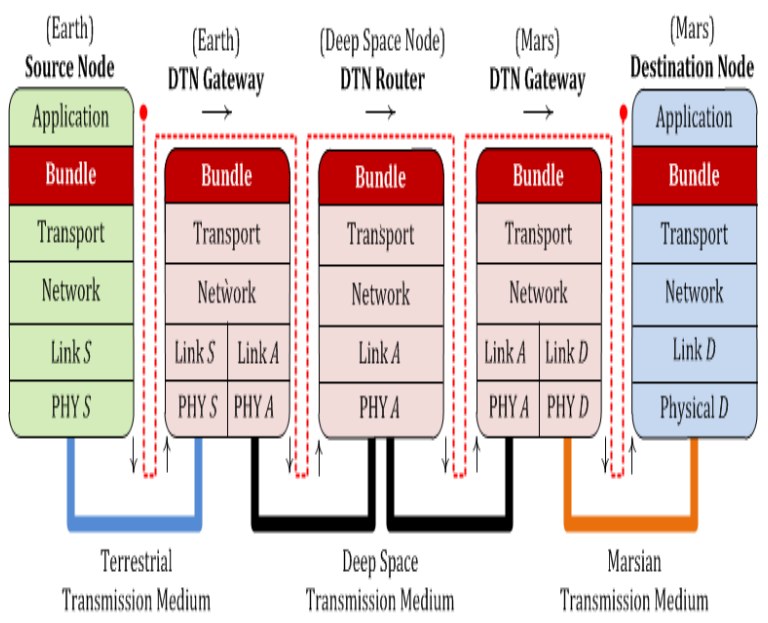

Fig 4: DTN Protocol Stack Communication Diagram

As shown in figure, layers underlying the bundle layer are network specific. Their existence shows that the bundle protocol by itself is not sufficient to carry information across the DTN but it relies on a variety of delivery protocol. These layers are referred as a convergence layer and are equipped with a set of protocol specific convergence layer adopter that are responsible for guiding DTN bundles through their corresponding protocols different procedure.

The network architecture for delay tolerant network consists of three major entities:

Host- The host is used to send or receive data in forms of the bundle.

Router- The router is used to forwards bundles to another node in a single region. It needs some buffer space because of uncertainty regarding future communication opportunity, for this it needs to store data for retransmission.

Gateway- The gateway is used for forwards bundles to nodes in different regions. It must supports interoperability, and are also used for performing authentication.

\section{MESSAGE TRANSMISSION AND BUNDLE LAYER}

The main problem in intermittently connected network is how to transport data from one region to another region through those IPN gateways. All internet protocol should terminate at IPN gateway and the message should be reencapsulated in a suitable format. Thus we need to develop a new protocol that can be able to accomplish this task.

New protocols must be able to account variable delays and the asymmetric bandwidth of the extra-terrestrial world and it must support non conversational communication.
One more significant problem is a bandwidth mismatch in between extraterrestrial and terrestrial network. For example throughput ranges from $1600 \mathrm{bps}$ and $250 \mathrm{Kbps}$ in case of earth to mars communication [12] while its ranges is in Mbps to Gbps for terrestrial network. Also due to resource constraints of terrestrial network, immutable propagation delays as well as additional overhead of queuing and scheduling delays occurs. Here queuing is required for data (arriving as well as those that needs retransmission due to failure). Also we need a proper tracking mechanism to impose an interactive session. The above mentioned all points are not only for the interplanetary network but it is also applicable for a general DTN. Also it is clear that at DTN gateway we need we need significant modification in a traditional internet protocol [9].

In delay tolerant network the messages are broken down into small packets known as Bundles. Here all information, data and control signals are contained in a single entity. These bundles are sent over an underlying network like an IP packet. Each bundle consists of the destination address, where the packet is destined to. The bundle also contains some data and metadata (data about data) along with some routing information. When messages are sent, it requires more capability for acknowledgement of sent data, as DTN suffers from lack of continuous connectivity. To enable this task up to some extant, the bundle layer is used to facilitate some of general purposes DTN services like:

Custody Transfer: It facilitates the sharing of acknowledgement of custodial acceptance in hop to hop basis.

Return Receipt: It enables the return confirmation facility that the bundle is received to the sending node.

Custody-Transfer Notification: Here the message is sent to the reply-to node regarding the change in custody of the communication

Bundle-Forwarding Notification: By this facility information is sent to the reply-to node or source node whenever the bundle is being forwarded to the next node

Priority of Delivery: Here the importance/ priority of the bundle can be set to Normal, Expedited or Bulk.

Authentication: This field is required for the purpose to verify the integrity of the message as well as the origin of the source.

Now we will consider the basics of the bundle protocol as given below:

\subsection{Name, Address and Binding process}

The nodes in a DTN network are identified by the end point identifier which are of maximum length 1024 byte. Here End Point Identifier (EID) [17] may represent a single node or a group of node. DTN relies on the concept that nodes are used to carry bundles from one region to another region. A node initially in a region, register itself as a part of that region and obtain an address. As this address is highly subject to change because of continuous change in the region of that particular node, but the name remains fixed irrespective of region. The late binding principals are used to map the address that are closest to the possible destination.

\subsection{Timing and Synchronization}

As in DTN end nodes are not always contactable, in this context synchronization $[9,10]$ might be a cakewalk: by using a synchronized clock. It is not simple but, one of the most 
critical issues. In addition to this timing [11] is required for tracking down expired bundle. For this purpose bundle creation timestamp field and the lifetime of bundle header are used.

\subsection{Reporting}

For a good reporting mechanism there should be an EID field (e.g. Report-to) in the bundle's header which indicate the nodes to which reports should be sent when required [18]. The bundle protocol specification differentiates between five type reports as listed below:

Table 1. Bundle Report Types

\begin{tabular}{|c|c|}
\hline Report Flag & Report Description \\
\hline $\begin{array}{c}\text { Custody } \\
\text { Accepted }\end{array}$ & $\begin{array}{c}\text { Sent by a candidate custodian after } \\
\text { accepting the custody transfer of a } \\
\text { bundle. }\end{array}$ \\
\hline Bundle Received & $\begin{array}{c}\text { Sent after bundle has arrived at the } \\
\text { destination node. }\end{array}$ \\
\hline $\begin{array}{c}\text { Bundle } \\
\text { Forwarded }\end{array}$ & $\begin{array}{c}\text { Sent after forwarding a particular } \\
\text { bundle. }\end{array}$ \\
\hline Bundle Delivered & $\begin{array}{c}\text { Sent after a successful bundle's } \\
\text { delivery by the Bundle Protocol to the } \\
\text { application. }\end{array}$ \\
\hline Bundle Deleted & $\begin{array}{c}\text { Sent at the time when an expired } \\
\text { bundle is deleted. }\end{array}$ \\
\hline
\end{tabular}

To request a particular type of report, its corresponding flag in the bundle's header is set. Also to avoid the possibility of infinite report the specifications explicitly specify clearly that no reports can be generated as results of other reports.

\subsection{Contact and Connection types}

DTN can be represented by an abstract graph G (v,e) where v is the set of nodes and $\mathrm{e}$ is the directed edges as shown below. The existence of edge e shows the ability of communication. It may be positive or zero or may fluctuate. When no communication is possible over the link / edge e, the capacity $\mathrm{Ce}(t)$, can be considered as a zero for this link.

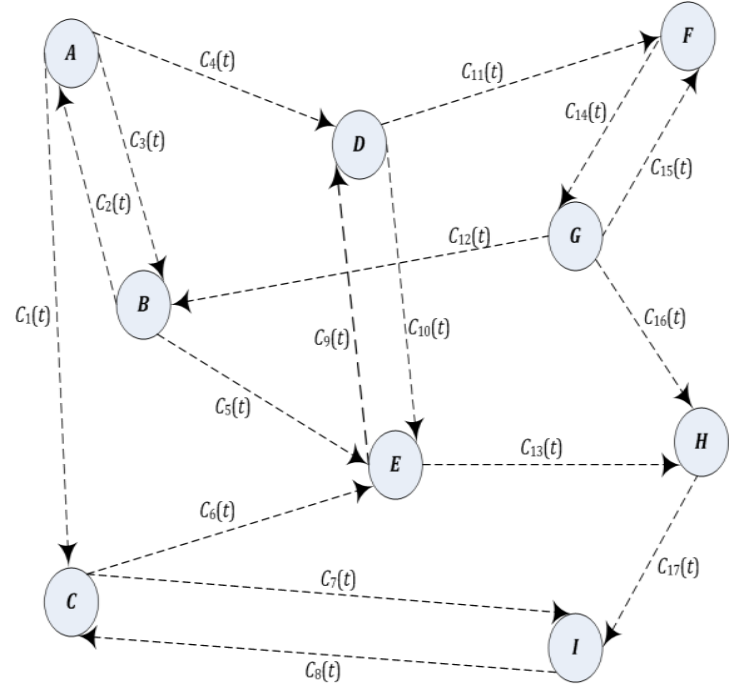

Fig 5: DTN Graph Abstraction

Thus the contact [13] may be defined as the time interval during which a time varying capacity $C e(t)$ of a edge "e" is positive.
Following above not only contact type changes but also connection type changes as described in [15].

a) Persistent Connection: It is a type of connection that is always available.

b) On Demand Connection: A connection that is initiated on request and become persistent till termination.

c) Scheduled Connection: A connection that is initiated at a particular time for a particular duration.

d) Opportunistic Connection: It is a type of connection that is established by unexpected opportunity.

e) Predicted Connection: A connection that can be predicted based upon some statically available information that provide sufficient confidence.

\subsection{Fragmentation}

As the DTN bundle travel through heterogeneous regions, that region may have the limitations on the maximum size of their respective PDU (protocol data unit). Also whenever the channel capacity is too low the transmission of large bundle may be impractical. The above two reasons may support regarding the requirement of fragmentation of the bundle.

Thus two types of fragmentation can be defined for DTN [2]:

\subsubsection{Proactive Fragmentation}

Here the large bundle may be subdivided into smaller size fragments before the establishment of connections. This fragmentation can be occurs only if we have prior knowledge of connection duration and channel capacity.

\subsubsection{Reactive Fragmentation}

In reactive fragmentation, subdivision of the large bundle takes place and smaller size fragments are created whenever it is indicated by the lower layer protocol that the larger size bundle has only partially transmitted.

Thus it is clear above that reactive fragmentation is more challenging to handle as it is dynamic fragmentation and it conflict with other custody transfer mechanism.

\subsection{Congestion and Flow Control}

Flow control can be defined as a mechanism that enforces limitation on the sending rate of a DTN forwarder and Congestion control is a mechanism used for handling the contention for persistent storage/buffer at a DTN node [15]. The authors of [14] propose definitions regarding congestion and flow control approaches. For flow control mechanism DTN relies on the implemented mechanism of underlying region-specific transport protocols such as TCP, X.25, Ready/Clear To Send (RTS/CTS) etc. A DTN node assumes the presence of flow control mechanisms for the purpose to ensure reliable message delivery.

Congestion control, however, is a much more complicated process due to the following reasons. One reason is Unpredictable availability of near future contacts which results accumulations of bundles in nodes' buffers and the other is Bundles stored till their expiration or the occurrence of an unusual event that cause deletion.

To avoid congestion, any of the following solutions as proposed in [2] may be used, such as unexpired bundles can be discarded or bundle can be displaced if enough storage capacity is available at nearby suitable nodes or a node may not accept more custody for incoming bundles. 


\section{CONCLUSION}

The major challenge in delay tolerant network is to enable efficient communication paradigm for intermittently connected environment or partition based network where the nodes are sparsely distributed. In this paper we have try to give emphasis on architectural aspects of delay tolerant network. We have first discussed about the architectural overview and bundle protocol specification of the delay tolerant network. The requirement of new layer"bundle layer" is also justified along with there naming and addressing mechanism. We have also given emphasis on contact and connection types as well as described types of fragmentation applicable in delay tolerant network. Timing and synchronization issue has also been discussed.

\section{REFERENCES}

[1] K. Fall, "A Delay-Tolerant Network Architecture for Challenged Internets," in Proc. ACM SIGCOMM Conf., pp. 27-34, Aug. 2003.

[2] K. Fall, S. Farrell, "DTN: An Architectural Retrospective," IEEE J. Sel. Areas Commun., Vol. 26, No. 5, June 2008.

[3] A. McMahon and S. Farrell, "Delay- and DisruptionTolerant Networking," IEEE Internet Computing, vol. 13, no. 6, pp. 82-87, Nov.2009.

[4] Delay-Tolerant Networking Research Group (DTNRG),http://www.dtnrg.org

[5] M. Demmer, "A Delay Tolerant Networking and System Architecture for Developing Regions," PhD. Dissertation, University of California, Berkeley, 2008.

[6] D. L. Mills, "Timekeeping in the Interplanetary Internet," University of Delaware, Available Online: http://www.ee.udel.edu/ mills/database/brief/ipin/ipin.p df, August 2004.
[7] A. Pentland, R. Fletcher, and A. Hasson, "DakNet: Rethinking Connectivity in Developing Nations," Computer, vol. 37, no. 1, pp. 78-83, Jan. 2004.

[8] K. Scott, S. Burleigh, "Bundle Protocol Specification," IETF Network Working Group, RFC5050, http://www.ietf.org/rfc/rfc5050.txt, November 2007.

[9] L. Wood, W. M. Eddy and P. Holliday, "A Bundle of Problems," IEEE Aerospace Conference, Big Sky, Montana, March 2009.

[10] J. Jackson, “The Interplanetary Internet," IEEE Spectrum, Volume 42, Issue No. 8, p. 30, August 2005.

[11] W. M. Eddy, "DTN Time Sync Issues," an e-mail to the IRTF dtninterest mailing list and following discussions, April 2008.

[12] V. Cerf, S. Burleigh, A. Hooke, L. Torgerson, R. Durst, K. Scott, E. Travis and H. Weiss, "Interplanetary Internet (IPN): Architectural Definition," Available Online: http://www.ipnsig.org/reports/memo-ipnrg-arch-00.pdf.

[13] S. Farrell and V. Cahill, "Delay- and Disruption-Tolerant Networking," Artech House INC., Foreword p. ix, 2006.

[14] V. Cerf, S. Burleigh, A. Hook, L. Torgerson, R. Dust, K. Scott, K. Fall and H. Weiss, "Delay-Tolerant Networking Architecture," IETF Network Working Group, RFC4838, Informational, http://www.ietf. org/rfc/rfc4838.txt, April 2007.

[15] The ZebraNet Wildlife Tracker, http://www.princeton.edu/ mrm/zebranet.html

[16] J. F. Kurose and K. W. Ross, "Computer Networking: A Top Down Approach," 5th Edition, Addison-Wesley, Pearsons Education INC., One Lake Street, Upper Saddle River, NJ 07458, 2010. 